\title{
USO DA ACUPUNTURA NO MANEJO DA DOR EM PACIENTES COM ALTERAÇÕES NA ARTICULAÇÃO TEMPOROMANDIBULAR (ATM).
}

\section{USE OF ACUPUNCTURE IN THE MANAGEMENT OF THE PAIN IN PATIENTS WITH CHANGES IN TEMPOROMANDIBULAR JOINT.}

\author{
Vera Lucia Rasera Zotelli* \\ Maria Paula Maciel Rando Meirelles* \\ Maria da Luz Rosário de Sousa"*
}

\begin{abstract}
RESUMO
A acupuntura, técnica bastante difundida devido às suas propriedades antiinflamatórias, ansiolíticas, miorelaxantes e ativadoras da função imunológica no organismo humano, tem sido muito utilizada como terapia coadjuvante em diversas especialidades odontológicas. O objetivo deste trabalho é apresentar o caso clínico de uma paciente com Disfunção Temporomandibular (DTM), tratada com acupuntura, e os resultados obtidos por essa técnica terapêutica no serviço odontológico da Faculdade de Odontologia de Piracicaba (FOP-UNICAMP). A paciente VASN, 34 anos, com queixa de dor na ATM, mais intensa no lado direito, com diagnóstico clínico de DTM e bruxismo, foi submetida ao tratamento por acupuntura. De acordo com a MTC (Medicina Tradicional Chinesa), foi encontrado o padrão de desequilíbrio energético da mesma e foi estabelecido um protocolo de tratamento que se demonstrou efetivo para redução dos sintomas.
\end{abstract}

DESCRITORES: Síndrome da disfunção temporomandibular • Acunpuntura • Dor facial

\section{ABSTRACT}

Acupuncture has increasingly been used as a complementary therapy in tooh treatment for its widely acknowledged properties: antiflamatory, ansiolitic, miorelaxing and as a stimulator of the human immunity system. The aim of this paper is to report a study case with the results of a temporomandibular joint (TMD) disorder treated with acupuncture in the Dental Service Department at the Dental School of Piracicaba (FOP- UNICAMP). The 34-years-old female patient was diagnosed with TMD, more intense on the right side of her face, and bruxism. The patient was taken to acupuncture treatment, according to the Traditional Chinese Medicine, and it was found a pattern of energetic imbalance. The protocol of treatment in this therapy was effective to reduce symptoms.

DESCRIPTORS: Temporomandibular joint disfunction • Acupuncture • Facial pain.

\footnotetext{
* Especialista em Periodontia e estagiária da Clínica de Acupuntura da Faculdade de Odontologia de Piracicaba da Universidade de Campinas - UNICAMP. Email: verarz@terra.com.br

** Mestre em Odontologia, Área de Concentração Cariologia. Email: mpaula_rando@yahoo.com.br

*** Professora Titular do Departamento de Odontologia Social da Faculdade de Odontologia de Piracicaba - UNICAMP. Coordenadora do Curso de Acupuntura em Odontologia. Email: luzsousa@fop.unicamp.br
} 
ZOTELLI VLR, RANDO-MEIRELLES $M P$,

SOUSA MLR.

USO DA ACU-

PUNTURA NO

MANEJO DA DOR

EM PACIENTES

COM ALTERAÇÕES

NA ARTICULAÇÃO

TEMPOROMANDIBU$\angle A R$ (ATM).

\section{6}

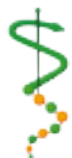

REVISTA DE ODONTOLOGIA DA

UNI VERS I DADE

CIDADE DE SÃO

PAULO

$2010 ; 22(2):$ $185-8, \quad M A I-A G 0$

\section{IN TRODUÇÃo}

A acupuntura tem se mostrado uma excelente alternativa terapêutica para o tratamento da DTM (Disfunção Temporomandibular). DTM é a denominação dada ao conjunto de distúrbios articulares e musculares que ocorrem na ATM (Articulação Tempormandibular) e região orofacial. A DTM tem etiologia multifatorial ${ }^{1,2}$.

Hábitos parafuncionais (apertar ou ranger os dentes), iatrogenias, hábitos de postura, além de fatores comportamentais, sociais e emocionais, podem predispor, iniciar, perpetuar ou resultar em uma disfunção temporomandibular ${ }^{3}$.

Para a acupuntura, a doença é resultado da interação entre os agentes agressores e a resposta do organismo, comandada pelo sistema nervoso central. Além disso, este sistema sofre a influência do corpo como um todo. Se o corpo estiver enfraquecido, em estado depressivo, sofrendo com ansiedades, isso se refletirá negativamente sobre o sistema nervoso central ${ }^{4}$.

No caso das lesões articulares, como, por exemplo, na ATM, se o espasmo muscular ao redor for demasiado, poderá levar à isquemia e a um círculo inflamatório vicioso, que levará a uma disfunção temporomandibular, gerando uma dor facial crônica ${ }^{4}$.

A acupuntura não visa tratar apenas o local comprometido do corpo, mas age sobre todo o sistema nervoso, estimulando o mecanismo de compensação e equilíbrio em todo o corpo. O mecanismo de ação da acupuntura no tratamento da DTM ocorre a partir da estimulação de pontos específicos do organismo (acupontos) através da punção com agulhas muito finas ${ }^{4}$.

Os pontos de acupuntura são considerados na MTC (Medicina Tradicional Chinesa), a área mais externa do corpo energético do indivíduo, funcionando como elo de comunicação entre o meio interno e externo. Alguns conceitos sobre os mecanismos de ação da acupuntura são importantes para se entender como ela pode atuar nos casos de DTM. A partir da estimulação de certos pontos, pode-se alterar a dinâmica da circulação sanguínea e também promover o relaxamento mus- cular, sanando o espasmo e diminuindo a inflamação e a dor. Além disso, a estimulação de alguns pontos pode promover a liberação de hormônios, como o cortisol e as endorfinas, promovendo a analgesia ${ }^{4}$.

Apesar de a Acupuntura produzir efeitos reais no tratamento de diversas doenças e muitos de seus efeitos terem sido esclarecidos pela medicina moderna, para se tratar uma doença, faz-se necessário chegar ao diagnóstico correto e individualizado, e estabelecer um esquema terapêutico adequado, que deverá ser realizado por um profissional competente ${ }^{4}$. Devido às suas inúmeras funções, tem sido utilizada como tratamento coadjuvante em diversas especialidades odontológicas.

Estudos têm demonstrado que a acupuntura vem contribuindo positivamente na prática odontológica ${ }^{5}$, seja da forma convencional, através da estimulação com agulhas, ou de forma mais moderna, por meio do uso de aparelhos de eletroestimulação ou lasers.

O objetivo deste trabalho é apresentar o caso clínico de uma paciente com Disfunção Temporomandibular (DTM), tratada com acupuntura, e os resultados por essa técnica terapêutica, no Serviço Odontológico da Faculdade de Odontologia de Piracicaba (FOP-UNICAMP).

\section{CASO CLÍNICO}

Paciente atendida no Serviço Odontológico da Faculdade de Odontologia de Piracicaba (FOP-UNICAMP). VASN, 34 anos, sexo feminino, farmacêutica, altura $1,62 \mathrm{~m}$ e peso $67 \mathrm{~kg}$. A paciente procurou a FOP queixando-se de dor na ATM, mais acentuada no lado direito e relatando ter bruxismo desde os 25 anos.

Previamente à aplicação de agulhas, foi realizada a anamnese e os exames de pulso e língua da paciente.

Durante a anamnese, a paciente relatou que tinha medo de dirigir, possuir "síndrome do pânico" e "distúrbio do sono" e que, para tais casos, toma medicação apropriada. Teve a primeira menstruação aos nove anos e atualmente sua menstruação é desregrada e tem TPM (tensão pré-menstrual). Disse ter se submetido a quatro inseminações artificiais e duas fertilizações sendo que na primeira vez ficou 
grávida de gêmeos e abortou e na segunda teve uma gravidez de alto risco da qual nasceu uma menina. Atualmente está fazendo mestrado e sente-se muito estressada.

$\mathrm{Na}$ anamnese segundo a MTC, a paciente relatou preferir calor, ter transpiração excessiva no tórax e na cabeça, sentir muita sede e beber mais refrigerantes do que água; preferir alimentos salgados, porém, sente muita necessidade de chocolate; ter sono leve; ter grande produção de cera no ouvido; retenção de líquidos; urina pouca e de cor escura e com odor forte; ser obstipada. A tez era amarelada, oleosa e porosa com cicatrizes de acne, manchas e sardas; a língua possuía marcas de dentes nas laterais, saburra amarela no centro e ponta avermelhada; pulso médio, rápido e escorregadio, lábios e gengivas normais. Declarou-se ser ansiosa, perfeccionista, preocupada e imediatista.

De acordo com os dados obtidos na anamnese, o diagnóstico estabelecido pela MTC foi de Deficiência de Yang do Baço-pâncreas.

Na sessão seguinte, foi iniciado o tratamento pela acupuntura. A paciente foi tratada buscando-se eliminar o excesso de mucosidade do baço-pâncreas. Os atendimentos foram realizados com aplicação de acupuntura sistêmica e auricular, em sessões semanais de 20 minutos. Foram usadas agulhas descartáveis de calibre $0.25 \times 25 \mathrm{~mm}$ para as punções e sementes para a auriculoterapia.

Foi seguido um protocolo preestabelecido para alterações de BP (baço-pâncreas) com agulhamento dos pontos VC12; TA3; IG4; BP4 e VG15 em todas as sessões. Os pontos auriculares estimulados com sementes foram Shen-men, coração, neurastenia e ATM e a paciente foi orientada a retirá-las após quatro dias.

Após as sessões, a paciente observou melhora da dor na região da articulação e relatou também ter tido mais coragem para enfrentar o seu medo de dirigir seu veículo.

\section{I SCUSSÃO}

A acupuntura tem apresentado bons resultados no tratamento de casos de dores crônicas, como no caso da DTM, devido às suas propriedades antiinflamatória, ansiolítica, mio-relaxante e ativadora da função imunológica no organismo humano.

Nesse caso clínico, a paciente procurou o tratamento por apresentar dor na região da ATM e porque tinha consciência de que apertava e rangia os dentes. A paciente estava com as emoções exacerbadas devido às várias tentativas para engravidar, e também submetida a stress devido à conclusão do seu título de mestrado. Esses fatores agravavam o apertamento dentário e aumentavam as dores na região da articulação temporomandibular.

Na MTC, a Síndrome de Deficiência indica fraqueza do organismo e de seu sistema de defesa ou desgaste, decorrente de doença prolongada ${ }^{6}$. Os sintomas de insônia, preocupação, palidez e oleosidade da tez apresentados pela paciente, associados à presença de saburra amarela e marcas de dentes na língua, levaram ao diagnóstico de baixa energia de Yang no baço-pâncreas.

A anamnese realizada seguindo os princípios da MTC possibilita um diagnóstico individualizado de acordo com o padrão energético em desarmonia, ou seja, pacientes com sinais e sintomas semelhantes recebem tratamentos diferenciados, pois a causa da dor é de origem diferente em cada paciente. De acordo com o atendimento realizado na Clínica de Acupuntura da Faculdade de Odontologia de Piracicaba - UNICAMP, existem sete protocolos diferentes para o controle da dor orofacial desencadeada por distúrbios temporomandibulares.

A estimulação de pontos do corpo, com propriedades para atuar no órgão doente, restabeleceu o equilíbrio energético do organismo e, assim, alcançou os resultados terapêuticos.

A MTC tem uma visão holística do ser humano, sendo assim, há uma interação entre todos os órgãos e estruturas e com as emoções do indivíduo. Assim, o paciente deve ser tratado como um todo para que ocorra a melhora de um sintoma localizado.

\section{CONCLUSÃO}

A paciente relatou redução dos sistemas dolorosos e melhora na qualidade do
ZOTELLI VLR, RANDO-MEIRELLES $M P$,

SOUSA MLR.

USO DA ACUPUNTURA NO MANEJO DA DOR EM PACIENTES COM ALTERAÇÕES NA ARTICULAÇÃO TEMPOROMANDIBU LAR (ATM).
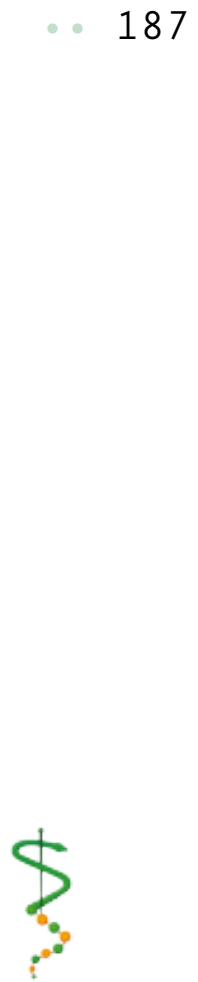

REVISTA DE ODONTOLOGIA DA UN I VERS I DADE Cidade de São PAULO

2010; 22(2): 185-8, MAI - AGO 
ZOTELLI VLR, RANDO-MEIRELLES $M P$, SOUSA MLR.

USO DA ACU-

PUNTURA NO MANEJO DA DOR EM PACIENTES COM ALTERAÇÕES NA ARTICULAÇÃO TEMPOROMANDIBULAR (ATM). sono.

Conclui-se que o uso da acupuntura como terapia integrativa em Odontologia em pacientes portadores de DTM tem se mostrado uma ferramenta útil, eficaz, de baixo custo e que proporciona uma meIhor qualidade de vida aos pacientes tra- tados.

\section{AGRADECIMENTOS}

Ao Dr. Jou Eel Jia e ao Dr. Jorge Eiji Sato pelo auxílio na elaboração dos protocolos de acupuntura para tratamento de dores orofaciais.

\section{REFERÊNCIAS}

1. DeBoever JA, Carlson GE. Etiologia e diagnóstico diferencial. In: Zarb GA. Disfunções da articulação temporomandibular e dos músculos da mastigação. São Paulo: Santos, 2000 p.171-7.

2. Barros JJ, Rode SM. Tratamento das disfunções craniomandibulares; ATM. São Paulo: Santos, 1995.

3. Rugh JD, Dahlström L. Mecanismos psicológicos e comportamentais. In: Zarb GA, Carlson GE. Disfunções da articulação temporomandibular e dos músculos da mastigação. São Paulo: Santos, 2000 p.208-17.

4 - Wen, Tom Sintan. Acupuntura clássica chinesa. São Paulo: Cultrix, 2006 p. 15-17.

5. Branco CA. Fonseca RB, Oliveira TRC, Gomes VL, Fernandes Neto AJ. Acupuncture as a complementary treatment option to temporomandibular dysfunction: review of the literature. Rev Odontol UNESP 2005; 34(1): 11-6.

6. Yamamura Y. Acupuntura tradicional: a arte de inserir, $2^{\mathrm{a}}$ ed .São Paulo: Roca; 2001.

Recebido em: 11/03/2010

Aceito em: 14/04/2010 